\title{
Mechanisms underlying brain monitoring during anesthesia: limitations, possible improvements, and perspectives
}

\author{
Marco Cascella \\ Department of Anesthesia, Endoscopy and Cardiology, National Cancer Institute 'G Pascale' Foundation, Naples, \\ Italy
}

\begin{abstract}
Currently, anesthesiologists use clinical parameters to directly measure the depth of anesthesia (DoA). This clinical standard of monitoring is often combined with brain monitoring for better assessment of the hypnotic component of anesthesia. Brain monitoring devices provide indices allowing for an immediate assessment of the impact of anesthetics on consciousness. However, questions remain regarding the mechanisms underpinning these indices of hypnosis. By briefly describing current knowledge of the brain's electrical activity during general anesthesia, as well as the operating principles of DoA monitors, the aim of this work is to simplify our understanding of the mathematical processes that allow for translation of complex patterns of brain electrical activity into dimensionless indices. This is a challenging task because mathematical concepts appear remote from clinical practice. Moreover, most DoA algorithms are proprietary algorithms and the difficulty of exploring the inner workings of mathematical models represents an obstacle to accurate simplification. The limitations of current DoA monitors - and the possibility for improvement - as well as perspectives on brain monitoring derived from recent research on corticocortical connectivity and communication are also discussed.
\end{abstract}

Key Words: Electroencephalography, Intraoperative awareness, Intraoperative neurophysiological monitoring.

\section{Introduction}

General anesthesia (GA) is a temporary, medically induced condition that includes loss of consciousness (LOC), amnesia, analgesia, relaxation of skeletal muscles, and loss of reflexes in the autonomic nervous system. Despite substantial advances in

Corresponding author: Marco Cascella, M.D.

Department of Anesthesia, Endoscopy and Cardiology, National Cancer Institute 'G Pascale' Foundation, Via Mariano Semmola, 162, Naples 80131, Italy

Tel: 39-081-5903586, Fax: 39-081-5903778

E-mail: m.cascella@istitutotumori.na.it

Received: November 16, 2015.

Revised: December 13, 2015.

Accepted: December 31, 2015.

Korean J Anesthesiol 2016 April 69(2): 113-120

http://dx.doi.org/10.4097/kjae.2016.69.2.113 our understanding of both neuroscientific and pharmacodynamic aspects of anesthetics, we do not yet have an overall picture of what happens to the brain during GA [1]. Consequently, anesthesiologists often refer to landmarks to induce narcosis correctly, avoiding a deeper state of anesthesia that can cause postoperative cognitive disorders, other postoperative complications, morbidity [2], and sudden emergencies that carry an increased risk of anesthesia awareness (AA) $[3,4]$. These points of reference encompass clinical and instrumental parameters, including the monitoring of ventilator parameters and anesthesia gas delivery. Additionally, standard clinical monitoring is often combined with methods that can assess depth of anesthesia (DoA) for better assessment of the level of consciousness during anesthesia [5-7]. As GA encompasses amnesia, analgesia, and immobility, "depth of anesthesia" may appear as a vague term. In this review, only methods for monitoring hypnotic depth are discussed, while nociceptive monitoring methods, including the surgical pleth index, analgesia nociception index, pupillometry,

(c) This is an open-access article distributed under the terms of the Creative Commons Attribution Non-Commercial License (http://creativecommons.org/ licenses/by-nc/4.0/), which permits unrestricted non-commercial use, distribution, and reproduction in any medium, provided the original work is properly cited. 
and neuromuscular monitoring devices are not considered. Thus, in the present context, DoA refers to brain monitoring, and is used synonymously with "depth of hypnosis."

\section{Brain Electrical Activity during General Anesthesia}

GA is induced by administration of two types of general anesthetics: intravenous agents, such as etomidate, midazolam, propofol, thiopental and ketamine; and inhaled agents including nitrous oxide (the oldest of all anesthetics) and various halogenated agents such as halothane, desflurane, isoflurane, and sevoflurane, and xenon. Because all general anesthetic drugs are relatively apolar molecules, they are able to cross the bloodbrain barrier, interacting, as explained below, with specific ion channels that regulate synaptic transmission and membrane potentials in several target areas of the brain. The binding of an anesthetic to one or more targets involves alterations in neuronal activities through neuron hyperpolarisation, resulting in increased neural inhibition or decreased synaptic excitation. There does exist a general scheme for explaining the operating mechanism of anesthetics $[8,9]$.

The actions of a general anesthetic at the molecular level impact on the brain's electrical activity, causing specific changes in the electroencephalogram (EEG). During anesthesia, the administration of anesthetics produces distinct EEG patterns; as the level of GA deepens, there is a progressive increase in lowfrequency and high-amplitude activity. In his fascinating study, Brown showed that, during anesthesia, EEG patterns can be described in relation to three different periods: induction, maintenance, and emergence [10]. Before induction, the patient has a normal, active EEG, with prominent alpha activity $(10 \mathrm{~Hz})$. After inducing anesthesia, the patient may enter a state referred to as paradoxical excitation (similar to drunkenness), characterised by incoherent speech, euphoria or dysphoria, distorted time perception, depersonalisation, and an EEG pattern that shows an increase in beta activity $(13-25 \mathrm{~Hz})$ [11]. This phase ends with LOC, in which the patient shows unconsciousness, depression of brain-stem reflexes, no response to nociceptive stimuli, apnoea, and the need for cardiorespiratory and thermoregulatory support. During the maintenance phase of anesthesia, it is possible to observe several different EEG patterns, depending on the DoA. A light state of narcosis, often defined as light anesthesia, is characterised by a decrease in EEG beta activity $(13-30 \mathrm{~Hz})$ and an increase in both EEG alpha activity $(8-12 \mathrm{~Hz})$ and delta activity $(0-4 \mathrm{~Hz})$; this pattern is particularly evident with propofol use [12]. As the anesthesia state becomes deeper, beta activity decreases, whereas alpha and delta activities increase, especially in the anterior EEG leads relative to the posterior leads [13]. In this state, the EEG pattern resembles that seen in non-rapid eye movement (or slow-wave) sleep. Because this state represents the target unconscious state, surgery is usually performed during this phase. A further state of deep anesthesia status is characterised by an EEG pattern comprising flat periods interspersed with periods of alpha and beta activity. This pattern of activity, called burst suppression, occurs in pathological states and during deep anesthesia and represents an interesting field of study. For example, Kenny et al. [14] showed significant differences in the duration, amplitude, and power of burst-suppression patterns induced by two common general anesthetics, sevoflurane and propofol, suggesting that the neuronal circuits involved in burst-suppression generation may differ among anesthetics. As this state of GA deepens, the gap between periods of alpha activity lengthens, and the amplitudes of the alpha and beta activity decrease, until the most profound state of narcosis is reached in which the EEG becomes isoelectric [15]. As stated in the Introduction, GA induces a reversible LOC. Emergence from narcosis can be characterised as a passive process that depends on the amount of drug administered. Although EEG patterns proceed in approximately the reverse order, from several different maintenance period phases, it is possible to observe a progressive decrease in alpha power and increased peak alpha frequency before the return of responsiveness. However, emergence is an active process with a distinct neurobiology [16]. Moreover, as recently shown by Hight et al. [17] the classic EEG pattern of emergence is not the only pattern of emergence from deep anesthesia. Indeed, some patients show no obvious progressive EEG change until an abrupt recovery of responsiveness.

Not all anesthetics work according to these EEG patterns during induction, maintenance, and emergence from anesthesia. As described below, some drugs, like ketamine, induce a more complex pattern of brain electrical activity with specific EEG patterns. This represents one of the many limitations of DoA monitoring devices.

\section{Operating Principles of DoA Monitors: Basis for Algorithm Development}

The EEG reflects the combined synaptic activity of excitatory and inhibitory post-synaptic potentials generated by cortical neurons [18]. Since 1937, anesthetists have been aware that anesthetics produce significant changes in EEGs [19]. However, the complex waves of a raw EEG are not interpreted easily and several problems have limited the application of standard EEG monitoring to anesthesia; for example, EEG does not change in a linear or monotonic fashion with changing anesthetic depth, and not all anesthetic agents produce similar EEG patterns. Thus, there is a need for simple and reliable techniques and tools that could be practical for use in all operating rooms. Over several decades of research, the complex EEG signal has been dissected 
to extract its core component, with significant advances also made in interpretation of the information that it may contain. The increased speed, flexibility, and economy of digital circuits, as well as advances in computer hardware and signal-processing algorithms, have produced revolutionary changes in the field of signal processing. Thus, methods have been developed that compress, simplify, and display various 'processed' summaries of EEG data. Consequently, many of these algorithms have been applied in commercially available DoA devices. However, there exists a major limitation to the study of the various algorithms of DoA monitors; namely, that they are proprietary algorithms. Thus, it is often difficult to understand the mathematical processes involved.

In this review, the evolution of raw EEG analysis is discussed as an introduction to this complex issue. One significant step forward was the possibility of translating the analogue raw EEG signal into a digital signal, because this renders it possible to represent discrete points in time at which values are underpinned by a fixed, rather than continuous, resolution. That is, digital signals are quantised in time, unlike analogue signals, which vary smoothly from moment to moment. However, the process of analogue-to-digital translation inevitably leads to some loss of fidelity in the resulting digital signal. Thus, it is essential to transform the EEG signal spectrum for accurate data processing. One of the mathematical models used for this purpose is Fourier analysis (or Fourier transform) [20]. This process decomposes the EEG time series into a voltage-by-frequency spectral graph commonly called a 'power spectrum,' with 'power' being the square of the EEG magnitude, and magnitude being the integral average of the amplitude of the EEG signal, measured from (+) peak to $(-)$ peak, across the time or epoch sampled. The epoch length determines the frequency resolution of the Fourier, with a 1 -s epoch providing a $1 \mathrm{~Hz}$ resolution $( \pm 0.5 \mathrm{~Hz}$ resolution), and a 4 -s epoch providing a $1 / 4 \mathrm{~Hz}$, or $\pm 0.125 \mathrm{~Hz}$, resolution.

A Fourier transform can transform a set of signal values $\mathrm{x}\left(\mathrm{t}_{\mathrm{i}}\right)$ sampled at time moments $t_{i}$ within a signal sample into a set of an equal number of complex values $\mathrm{X}\left(\mathrm{f}_{\mathrm{i}}\right)$ corresponding to a set of frequencies $\mathrm{f}_{\mathrm{i}}$ :

$$
X\left(f_{i}\right)=\sum_{t_{i}} x\left(t_{i}\right) e^{-i 2 \pi f_{i} t_{i}}
$$

A Fourier analysis generates a frequency spectrum $X\left(f_{i}\right)$, which is simply a histogram of amplitudes or phase angles as a function of the frequency of the signal $\mathrm{x}\left(\mathrm{t}_{\mathrm{i}}\right)$. As anesthesiologists are clinicians, and therefore may not be familiar with biostatistics, explaining this complex transformation is possible only by using a practical example. The concept may be illustrated in terms of the effect of passing a white light through a glass prism, thus creating a rainbow (or spectrum). Each color of light represents a unique frequency photon, and the relative brightness of the colors is a measure of the energy amplitude at each fre- quency [21].

Any measured signal, such as the spectral components $\mathrm{X}\left(\mathrm{f}_{\mathrm{i}}\right)$, transformed by the Fourier technique into the frequency domain will have both an amplitude and a phase component for each harmonic frequency. Nevertheless, this basilar approach to transforming and processing the raw signal is laborious. Thus, processing commonly uses specific algorithms, such as the fast Fourier transform $[22,23]$.

Fourier analysis was improved by using bispectrum measures, also called bispectral (BIS) analysis. While the algorithms of Fourier analysis lend themselves to measurement of the phase of component frequencies relative to the start of the epoch, BIS analyses allow for phase correlation between different frequency components. Moreover, BIS analysis has several additional characteristics that may be advantageous for processing EEG signals. For example, suppression of sources of noise involves significant enhancement of the signal. Technically, the bispectrum quantifies the relationships among the underlying sinusoidal components of the EEG, and examines the relationship between the sinusoids at two primary frequencies, $\mathrm{f} 1$ and $\mathrm{f} 2$, and a modulation component at the frequency $\mathrm{f} 1+\mathrm{f} 2$. This set of three frequency components ( $\mathrm{f} 1, \mathrm{f} 2$, and $\mathrm{f} 1+\mathrm{f} 2$ ) is known as a triplet. For each triplet (i.e. the bispectrum), it is possible to precisely calculate the amount incorporating both phase and power information, such that the bispectrum is a statistic used to find evidence of non-linear interactions in signals.

Based on these assumptions, all commercial DoA monitors analyse and quantify spontaneous or evoked raw EEG data using mathematical methods. Accordingly, and also by using specific algorithms, these devices provide several indices allowing for an easier reading of anesthesia status. These dimensionless indices are constructed, abstract quantities not directly linked to any physiological parameter. Among their other features, they are characterised by inherent time delays, consistent with the processing time, and the calculation and removal, through specific algorithms, of certain artefacts.

\section{Depth of Anesthesia Monitors and Indices}

In 1994, Sigl and Chamoun [24] were the first to describe the use of BIS technology for brain monitoring. Their BIS system includes a non-invasive adhesive sensor, a patient interface cable, a digital signal converter, a BIS engine (microprocessor), and a monitor. The technology is also available as a module that can be integrated into other manufacturers' monitoring systems.

As noted previously, raw EEG signals can be difficult to interpret; thus, the BIS engine processes EEG data from a singlechannel EEG signal, measured from the patient's forehead according to an algorithm that combines select EEG features to produce a single dimensionless number, which is the 'BIS 
index.' The BIS algorithm is not publicly available. We know that this value is a statistically-based, empirically-derived, complex parameter equivalent to the weighted sum of several EEG subparameters, including a time domain, a frequency domain, and several high-order spectral subparameters [25]. The BIS index ranges from 0 (equivalent to EEG silence) to 100. A BIS value between 40 and 60 indicates an appropriate level for GA with a low probability of consciousness. In addition to the BIS index, the device displays other data, such as the BIS trend, which is a graphic representation of BIS index values over time, the signal quality index (SQI) bar, and the suppression ratio (SR). While SQI measures the reliability of the signal (a higher SQI number indicates more reliable BIS values), the SR number is the percentage of time over the last 63-s during which the EEG signal was suppressed (flat-lined). The monitor also displays a single-channel EEG waveform and an electromyography (EMG) bar graph, which, similar to the SQI, is used to help determine whether the BIS index values are reliable.

The BIS monitor was the first EEG-based DoA monitor. It is the most widely used system for assessing the brain monitoring of GA. Nevertheless, during the last 15-20 years, other EEGbased technologies have been studied [26], and some of them have become commercially available, such as E-Entropy and Narcotrend equipment (MonitorTechnik, Bad Bramstedt, Germany), and auditory evoked potential (AEP) measuring devices.

E-Entropy uses a proprietary algorithm to map the entropy of the EEG signal to the behavioural responses of the patients [27]. Here, the theoretical assumption is that irregularity in the EEG signal decreases under anesthesia. This irregularity can then be inferred by the entropy and used to estimate the DoA. The concept of spectral entropy originates from a measure called Shannon entropy [28], which is applied to the power EEG spectrum signal. The following steps are required to compute the spectral entropy for a particular epoch of the signal within a particular frequency range $[f 1, f 2]$. From the Fourier transform $X\left(f_{i}\right)$ of the signal $\mathrm{x}\left(\mathrm{t}_{\mathrm{i}}\right)$, the power spectrum $\mathrm{P}\left(\mathrm{f}_{\mathrm{i}}\right)$ is calculated by squaring the amplitudes of each element $\mathrm{X}\left(\mathrm{f}_{\mathrm{i}}\right)$ of the Fourier transform:

$$
P\left(f_{i}\right)=X\left(f_{i}\right) * X^{\wedge}\left(f_{i}\right)
$$

In this formula, $X^{\wedge}\left(f_{i}\right)$ is the complex conjugate of the Fourier component $\mathrm{X}\left(\mathrm{f}_{\mathrm{i}}\right)$. The next step of the algorithm is mathematical normalisation of the overall frequency range of values between 1 (maximum irregularity) and 0 (complete regularity). However, this transformation operates in parallel with the variable time, producing time-frequency-balanced spectral entropy in which the signal values $\mathrm{x}\left(\mathrm{t}_{\mathrm{i}}\right)$ are sampled within a finite time window (epoch) of a selected length with a particular sampling frequency. That is, to elaborate the signal rapidly, the algorithm allows for the study of each frequency in the context of an optimal time window.

Simplifying, through this algorithm, the degree of disorder of EEG and frontal EMG, the data are converted into two values that indicate the DoA. The first value, response entropy, provides an indication of the patient's responsiveness to external stimuli and signals early awakening. The second value, state entropy, is a stable parameter that may be used to assess the hypnotic effects of anesthetic agents on the brain. More ordered signals, with less variation in the wavelength and amplitude over time, produce higher entropy values and may indicate that the patient is awake. Regular signals, with a constant wavelength and amplitude over time, produce low or zero entropy values, indicating a low probability of AA recall and suppression of brain electrical activity. The response entropy scale ranges from 0 (no brain activity) to 100 (fully awake), and the state entropy scale ranges from 0 (no brain activity) to 91 (fully awake). The clinically relevant target range for response entropy is 40-60, while state entropy values near 40 indicate a low probability of consciousness.

The Narcotrend technology (MonitorTechnik), developed at the University Medical School of Hannover, Germany, analyses raw EEG data using spectral analysis to produce a number of parameters. Multivariate statistical methods using proprietary pattern recognition algorithms are applied to provide an automatically classified EEG signal on the basis of a visual assessment of the EEG, as related to Loomis' sleep classification system of 1937 [29]. The EEG visual classification scale ranges from stage A (awake) to stage F (very deep hypnosis), with stage E indicating the appropriate depth of anesthesia for surgery. Stages A-F were further subdivided into three sub-stages; thus, the Narcotrend Monitor can automatically distinguish between 14 or 15 stages (depending on the software version used). Also, the Narcotrend software includes a dimensionless Narcotrend index, ranging from 100 (awake) to 0 (electrical silence) [30].

Evoked potentials measure EEG responses to repetitive AEPS [31] or visual stimuli, and measure the integrity of the neural pathways that carry information from the periphery to the cortex. Here, the rationale is that while the BIS value is an index of hypnosis, evoked potentials show responses to stimuli. This is a significant translational field of study involving neurological, acoustic, and anesthesia data [32]. The AEP monitoring technique isolates the neurophysiological signal generated during stimulation of the eighth cranial nerve using a repetitive auditory stimulus (e.g. with a bilateral click stimulus of $70 \mathrm{~dB}$ intensity and $2 \mathrm{~ms}$ duration delivered through headphones [Alaris AEP Monitor; Alaris Medical Systems Inc., San Diego, CA, USA]). Repeated sampling allows the signal to be extracted from the background EEG noise. Furthermore, the signal is acquired using EEG electrodes located on the mastoid processes, a midline reference electrode, and a ground electrode. New AEP technologies are not only based on AEP, but also include spectral EEG 
parameters (AEP Monitor/2; Danmeter A/S, Odense, Denmark) that provide a dimensionless number; i.e. the composite AEPs index (cAAI), which uses both cortical EEG and AEP data [33]. This composite index (0-100 or $0-60)$ was considered to be a measure of the overall balance between noxious stimulation, analgesia, and hypnosis. Unlike the BIS index, the transition from asleep to awake is characterised by a sudden increase in the AEP index. However, these instruments have not yet gained wide popularity as DoA monitors, partly due to problems relating to signal interference. The main characteristics of the newest AEP devices are their small size and battery operation, such that the unit can travel with the patient from the induction room to the operating room, and on to the intensive care unit, enabling uninterrupted patient monitoring.

\section{Limitations in EEG Brain Monitoring, Possible Improvements, and Perspectives}

The BIS monitor was initially considered as the best tool for monitoring cerebral activity during anesthesia. Because the AA phenomenon has historically been the major concern in anesthesiology, it can be considered as the proverbial "philosopher's stone" of anesthesia monitoring. This signalled the start of a hard-fought battle on the pages of scientific journals [34]. The most recent BIS monitor algorithm (BIS XP ver. 4.0; Aspect Medical Systems, Newton, MA, USA) may produce lower BIS scores compared to older models for the same level of hypnosis. This difference is secondary to the inclusion, in more recent models, of mechanisms that reduce the level of noise, interference, and EMG activity, resulting in lower BIS values [35]. However, this advance in technology did not solve all of the problems related to the reliability of BIS monitoring. Indeed, the manufacturer of BIS recommends that BIS values should be interpreted cautiously in patients with known neurological disorders, and in those taking psychoactive medications. Moreover, BIS analysis is sensitive to changes in EMG activity; thus, the newer versions may also be ineffective for detecting a sudden change in the anesthesia status of patients receiving neuromuscular-blocking drugs [36].

Moreover, limitations affect other DoA monitors because of artefacts generated by the patient (e.g. due to eye movements and muscle activity) or external sources (poor skin contact, mains or power line interference, electrocautery), and clinical situations in which there is a lack of reliability. For example, EEntropy has been validated for use only in patients over the age of 2 years; it cannot be used for patients undergoing procedural or conscious sedation, and seizure activity may cause interference. In addition, E-Entropy readings may be inconsistent when monitoring patients with neurological disorders or in those on psychoactive medications. Although Narcotrend is equipped with artefact-detection algorithms, to exclude segments contaminated with artefacts, Narcotrend values should be interpreted cautiously in patients with a history of central nervous system disease.

Age is another underestimated variable in DoA monitoring. For example, under anesthesia, premature-born children display different EEG patterns versus term-born children [37], and the BIS index must be interpreted cautiously in the detection of depth of sedation in children [38] and elderly patients [39].

A significant limitation to actual DoA indices lies in their neurophysiological basis, because they assume that anesthetics induce slowing of the EEG oscillations with increasing doses. Thus, slower oscillations should clearly indicate a more profound state of GA. However, not all anesthetics show this EEG pattern. For example, ketamine shifts the alpha peaks of bicoherence induced by propofol to higher frequencies, perhaps through modulation of the non-linear sub-cortical reverberating network [40]. However, nitrous oxide suppresses low-frequency power, which can influence EEG monitoring parameters by increasing the value of the indices at clinically accepted doses [41].

This limitation seems to have been overcome by the development of devices that incorporate specific algorithms, designed in the early 2000s [42]. One of these algorithms is the patient state index (PSI), which is another index of the level of hypnosis/awareness. The PSI is calculated by a high-resolution fourchannel EEG monitor that collects information on frequency and phase brain electric activity according to anterior-posterior relationships in the brain, as well as coherence between bilateral brain regions. The PSI provides a continuous numeric value, derived from systematic studies of the complex of changes in brain state that were observed to reversibly accompany loss and return of consciousness independent of anesthetic class. Indeed, the variables selected for incorporation in the PSI displayed very significant heterogeneity of variance at different levels of sedation/hypnosis (sensitivity) but non-significant differences across anesthetic agents at a specified level (specificity) [43]. All these data suggest that technology using PSI (e.g. the SEDLine monitor; Hospira, Lake Forest, IL, USA) could be superior to other classic EEG monitors, such as the BIS. However, comparative studies showed that the PSI offered only a better correlation with the end-tidal concentration of desflurane, and PSI values were less affected by electrical interference, especially electrocautery. Although the PSI showed a better performance during both the induction of, and emergence from, GA during the maintenance period, the PSI values were comparable to those of the BIS [44]. Thus, the PSI is only a valid alternative to BIS monitors and does not completely solve the problem of gaps in brain monitoring during anesthesia.

Another branch of study concerns improvement of methods already in use, by improving their algorithms. As mentioned 
previously, a recent algorithm for the BIS monitor conferred reduced interference. Additionally, beyond response and state entropy, at least 10 entropy algorithms were investigated with respect to their ability to depth-monitor anesthesia and detect burst suppression. Although each entropy index showed advantages and disadvantages, the right combination of these indices could help improve current clinical indices for brain anesthesia monitoring [45].

However, the true limit to GA brain monitoring is that commercial brain monitoring devices do not accurately discriminate between consciousness and unconsciousness because their functioning is based on EEG signal analysis rather than consideration of corticocortical connectivity and communication [46]. This is a complex topic encompassing the operating mechanism of general anesthetics. Although we understand that the anesthetic effect is realised through ligand-receptor interactions in different brain areas, a major question remains regarding the physiological mechanisms by which anesthetics interfere with cortical and subcortical signals [41] arising, for example, from the thalamus. However, the thalamus is not central to the transition between conscious and unconscious states; more recent studies outline the role of cortical areas, such as the primary visual cortex [47] and the primary somatosensory cortex, which is a key area involved in the processing of nociceptive inputs [48] from the posterior cingulate cortex, precuneus, and mesial parietal cortex. These mesial cortical areas are functionally specialised regions of the human cerebral cortex, interconnected by a dense network of cortico-cortical axonal pathways. According to a modern neurofunctional approach, based on so-called cognitive brain architecture [49], these cortical areas arrange into both a structural and functional brain core, which has an important role in functional integration [50]. Nevertheless, the role of the thalamus is not secondary; rather, it acts as a relay in corticocortical communication, because all neocortical areas receive thalamic inputs. Indeed, some thalamocortical pathways relay information from ascending pathways (first-order thalamic relays) and others relay information from other cortical areas (higher-order thalamic relays). Thus, efficient communication among cortical areas requires thalamic integrity and thalamic lesions lead to functional disarray despite cortical activation [51].

According to this line of investigation, scientific research has enabled the development of new technologies for cerebral monitoring, and the studies of Boly et al. [52] on spectral EEG changes after propofol administration are very important. At the same time, Purdon et al. [53] also published their fascinating research explaining that the EEG pattern, and changes therein, are real-time indicators of the transition from consciousness to anesthesia. Transcranial magnetic stimulation (TMS) is a noninvasive method used both to stimulate small regions of the brain and to measure the activity and function of specific brain circuits in humans. Indeed, it is used to examine the integrity of the fast-conducting corticomotor pathways in a wide range of diseases associated with motor dysfunction [54]. The principles of this technique can be applied to the study of the relationship between consciousness and anesthesia. Recent studies have investigated the EEG response to TMS in subjects in diverse states of consciousness (e.g. wakeful rest, deep sleep, GA) [55,56], as well as in propofol-induced GA [57]. Thus, a technique that was created to investigate the dynamics of consciousness could have various other possible applications, including as a precise tool for brain monitoring during anesthesia.

Others authors have focused on building new algorithms, starting from the analysis of EEG signals. For example, Mirsadeghi et al. [58] proposed a new method for distinguishing between awake and anesthetised states. Their methodology is very interesting: through a specific analysis that uses a dimensionality reduction method (from high-dimensional to low-dimensional data), they processed some linear and non-linear features of raw EEG signals, citing an accuracy of $88.4 \%$ for classifying the EEG signal into conscious and unconscious states. More complex algorithms have been proposed to combine EEG features and hemodynamic variables for better classification of anesthesia status [59]. Schneider et al. [60] studied a combination of clinical standard monitoring, EEG, and patient and drug variables, demonstrating that it is possible to distinguish between different levels of anesthesia. However, their algorithm uses a complex analysis with a later construction, and is only marginally useful for rapid recognition of intraoperative awakening, particularly under conditions of neuromuscular blockade.

\section{Conclusions}

Several DoA monitors are now on the market; the technology underpinning these monitors is based on the results of studies of complex algorithms and mathematical models, related to the brain's electrical activity during GA. These devices began to be commercialised in the early 1990s and, because they offered an immediate means (in the form of various indices) of assessing the level of consciousness during anesthesia, there was a rapid increase in their use. Nevertheless, despite initial enthusiasm, the use of DoA monitors remains a major controversy in anesthesiology. Indeed, many factors, such as age, race, gender, low core body temperature, acid-base imbalances, low blood glucose, drugs administered to the patient (e.g. neuromuscular blocking agents), and brain ischaemia, have a significant effect on raw EEG data. Additionally, DoA monitors are limited by their calibration range and interpatient variability in doseresponse curves.

The study of signal transduction, from raw EEG data to an index, represents a hidden and fascinating mathematical world 
that is moving towards improvement of the reliability of indices of hypnosis. However, despite refinement of the algorithms, it is currently impossible to ameliorate the influence of all artefacts. What, then, are the current perspectives and goals? A better understanding of the transformation of cortical and subcorti- cal activity into EEG signals, as well as resolution of the puzzle concerning the link between consciousness and anesthesia with the processing of algorithms ad hoc, could allow for the development of DoA monitors for precise determination of the anesthesia status of patients during GA.

\section{References}

1. Hutt A, Hudetz AG. Editorial: General anesthesia: from theory to experiments. Front Syst Neurosci 2015; 9: 105.

2. Frost EA. Differential diagnosis of delayed awakening from general anesthesia: a review. Middle East J Anaesthesiol 2014; $22: 537-48$.

3. Pandit JJ, Andrade J, Bogod DG, Hitchman JM, Jonker WR, Lucas N, et al. 5th National Audit Project (NAP5) on accidental awareness during general anaesthesia: summary of main findings and risk factors. Br J Anaesth 2014; 113: 549-59.

4. Chung HS. Awareness and recall during general anesthesia. Korean J Anesthesiol 2014; 66: 339-45.

5. Musizza B, Ribaric S. Monitoring the depth of anaesthesia. Sensors (Basel) 2010; 10: 10896-935.

6. Palanca BJ, Mashour GA, Avidan MS. Processed electroencephalogram in depth of anesthesia monitoring. Curr Opin Anaesthesiol 2009; 22: 553-9.

7. Lobo FA, Schraag S. Limitations of anaesthesia depth monitoring. Curr Opin Anaesthesiol 2011; 24: 657-64.

8. Forman SA, Chin VA. General anesthetics and molecular mechanisms of unconsciousness. Int Anesthesiol Clin 2008; 46: 43-53.

9. Perouansky M. The quest for a unified model of anesthetic action: a century in Claude Bernard's shadow. Anesthesiology 2012; 117: 465-74.

10. Brown EN, Lydic R, Schiff ND. General anesthesia, sleep, and coma. N Engl J Med 2010; 363: 2638-50.

11. McCarthy MM, Brown EN, Kopell N. Potential network mechanisms mediating electroencephalographic beta rhythm changes during propofol-induced paradoxical excitation. J Neurosci 2008; 28: 13488-504.

12. Feshchenko VA, Veselis RA, Reinsel RA. Propofol-induced alpha rhythm. Neuropsychobiology 2004; 50: 257-66.

13. Tinker JH, Sharbrough FW, Michenfelder JD. Anterior shift of the dominant EEG rhythm during anesthesia in the Java monkey: correlation with anesthetic potency. Anesthesiology 1977; 46: 252-9.

14. Kenny JD, Westover MB, Ching S, Brown EN, Solt K. Propofol and sevoflurane induce distinct burst suppression patterns in rats. Front Syst Neurosci 2014; 8: 237.

15. Al-Kadi MI, Reaz MB, Ali MA. Evolution of electroencephalogram signal analysis techniques during anesthesia. Sensors (Basel) 2013; 13: 6605-35.

16. Kushikata T, Hirota K. Mechanisms of anesthetic emergence: evidence for active reanimation. Curr Anesthesiol Rep 2014; 4: 49-56.

17. Hight DF, Dadok VM, Szeri AJ, García PS, Voss L, Sleigh JW. Emergence from general anesthesia and the sleep-manifold. Front Syst Neurosci 2014; 8: 146.

18. Marchant N, Sanders R, Sleigh J, Vanhaudenhuyse A, Bruno MA, Brichant JF, et al. How electroencephalography serves the anesthesiologist. Clin EEG Neurosci 2014; 45: 22-32.

19. Gibbs FA, Gibbs EL, Lennox WG. Effect on the electroencephalogram of certain drugs which influence nervous activity. Arch Intern Med (Chic) 1937; 60: 154-66.

20. Castellanos NP, Makarov VA. Recovering EEG brain signals: artifact suppression with wavelet enhanced independent component analysis. J Neurosci Methods 2006; 158: 300-12.

21. Barlow JS. Artifact processing in EEG data processing, clinical applications of computer analysis of EEG and other neurophysiological signal. Edited by Lopes Da Silva FH, Storm van Leeuwen W, Remond A: Amsterdam, Elsevier. 1986, pp 15-62.

22. Sorensen HV, Jones DL, Heideman MT, Burrus CS. Real-valued fast Fourier transform algorithms. IEEE Trans Signal Process 1987; $35: 849-63$.

23. Cooley JW, Tukey JW. An algorithm for machine calculation of complex Fourier series. Math Computation 1965; 19: $297-301$.

24. Sigl JC, Chamoun NG. An introduction to bispectral analysis for the electroencephalogram. J Clin Monit 1994; 10: 392-404.

25. Rampil IJ. A primer for EEG signal processing in anesthesia. Anesthesiology 1998; 89: 980-1002.

26. Zikov T, Bibian S, Dumont GA, Huzmezan M, Ries CR. Quantifying cortical activity during general anesthesia using wavelet analysis. IEEE Trans Biomed Eng 2006; 53: 617-32.

27. Viertiö-Oja H, Maja V, Särkelä M, Talja P, Tenkanen N, Tolvanen-Laakso H, et al. Description of the Entropy algorithm as applied in the Datex-Ohmeda S/5 Entropy Module. Acta Anaesthesiol Scand 2004; 48: 154-61.

28. Shannon CE. A mathematical theory of communication. Bell System Techn J 1948; 27: 623-56.

29. Loomis AL, Harvey EN, Hobart CA. Cerebral states during sleep as studied by human brain potentials. J Exp Psychol 1937; 21: 127-44.

30. Kreuer S, Wilhelm W. The Narcotrend monitor. Best Pract Res Clin Anaesthesiol 2006; 20: 111-9.

31. Plourde G. Auditory evoked potentials. Best Pract Res Clin Anaesthesiol 2006; 20: 129-39.

32. McNeer RR, Bohórquez J, Ozdamar O. Influence of auditory stimulation rates on evoked potentials during general anesthesia: relation between the transient auditory middle-latency response and the 40-Hz auditory steady state response. Anesthesiology 2009; 110: 1026-35. 
33. Weber F, Zimmermann M, Bein T. The impact of acoustic stimulation on the AEP monitor/2 derived composite auditory evoked potential index under awake and anesthetized conditions. Anesth Analg 2005; 101: 435-9.

34. Kerssens C, Sebel PS. To BIS or not to BIS? That is the question. Anesth Analg 2006; 102: 380-2.

35. Duarte LT, Saraiva RA. When the bispectral index (Bis) can give false results. Rev Bras Anestesiol 2009; 59: 99-109.

36. Schuller PJ, Newell S, Strickland PA, Barry JJ. Response of bispectral index to neuromuscular block in awake volunteers. Br J Anaesth 2015; 115 Suppl 1: i95-i103.

37. Poorun R, Hartley C, Goksan S, Worley A, Boyd S, Cornelissen L, et al. Electroencephalography during general anaesthesia differs between term-born and premature-born children. Clin Neurophysiol 2016; 127: 1216-22.

38. Malviya S, Voepel-Lewis T, Tait AR, Watcha MF, Sadhasivam S, Friesen RH. Effect of age and sedative agent on the accuracy of bispectral index in detecting depth of sedation in children. Pediatrics 2007; 120: e461-70.

39. Yamashita K, Terao Y, Inadomi C, Takada M, Fukusaki M, Sumikawa K. Age-dependent relationship between bispectral index and sedation level. J Clin Anesth 2008; 20: 492-5.

40. Ching S, Cimenser A, Purdon PL, Brown EN, Kopell NJ. Thalamocortical model for a propofol-induced alpha-rhythm associated with loss of consciousness. Proc Natl Acad Sci U S A 2010; 107: 22665-70.

41. Mashour GA. Top-down mechanisms of anesthetic-induced unconsciousness. Front Syst Neurosci 2014; 8: 115.

42. Gugino LD, Chabot RJ, Prichep LS, John ER, Formanek V, Aglio LS. Quantitative EEG changes associated with loss and return of consciousness in healthy adult volunteers anaesthetized with propofol or sevoflurane. Br J Anaesth 2001; 87: 421-8.

43. Prichep LS, Gugino LD, John ER, Chabot RJ, Howard B, Merkin H, et al. The Patient State Index as an indicator of the level of hypnosis under general anaesthesia. Br J Anaesth 2004; 92: 393-9.

44. Chen X, Tang J, White PF, Wender RH, Ma H, Sloninsky A, et al. A comparison of patient state index and bispectral index values during the perioperative period. Anesth Analg 2002; 95: 1669-74.

45. Liang Z, Wang Y, Sun X, Li D, Voss LJ, Sleigh JW, et al. EEG entropy measures in anesthesia. Front Comput Neurosci 2015 ; 9: 16.

46. Mashour GA, Pryor KO. Consciousness, memory, and anesthesia. In: Miller's Anesthesia. 8th ed. Edited by Miller RD: Philadelphia, Churchill Livingstone. 2015, pp 287-9.

47. Leopold DA. Primary visual cortex: awareness and blindsight. Annu Rev Neurosci 2012; 35: 91-109.

48. Kuo CC, Lee JC, Chiou RJ, Tsai ML, Yen CT. Spatiotemporal changes of neuronal responses in the primary somatosensory cortex to Noxious tail stimulation in awake and pentobarbital-anesthetized rats. Chin J Physiol 2015; 58: 332-42.

49. Petersen SE, Sporns O. Brain networks and cognitive architectures. Neuron 2015; 88: 207-19.

50. Hagmann P, Cammoun L, Gigandet X, Meuli R, Honey CJ, Wedeen VJ, et al. Mapping the structural core of human cerebral cortex. PLoS Biol 2008; 6: e159.

51. Guillery RW, Sherman SM. Thalamic relay functions and their role in corticocortical communication: generalizations from the visual system. Neuron 2002; 33: 163-75.

52. Boly M, Moran R, Murphy M, Boveroux P, Bruno MA, Noirhomme Q, et al. Connectivity changes underlying spectral EEG changes during propofol-induced loss of consciousness. J Neurosci 2012; 32: 7082-90.

53. Purdon PL, Pierce ET, Mukamel EA, Prerau MJ, Walsh JL, Wong KF, et al. Electroencephalogram signatures of loss and recovery of consciousness from propofol. Proc Natl Acad Sci U S A 2013; 110: E1142-51.

54. Groppa S, Oliviero A, Eisen A, Quartarone A, Cohen LG, Mall V, et al. A practical guide to diagnostic transcranial magnetic stimulation: report of an IFCN committee. Clin Neurophysiol 2012; 123: 858-82.

55. Ferrarelli F, Massimini M, Sarasso S, Casali A, Riedner BA, Angelini G, et al. Breakdown in cortical effective connectivity during midazolam-induced loss of consciousness. Proc Natl Acad Sci U S A 2010; 107: 2681-6.

56. Sarasso S, Rosanova M, Casali AG, Casarotto S, Fecchio M, Boly M, et al. Quantifying cortical EEG responses to TMS in (un)consciousness. Clin EEG Neurosci 2014; 45: 40-9.

57. Schartner M, Seth A, Noirhomme Q, Boly M, Bruno MA, Laureys S, et al. Complexity of multi-dimensional spontaneous EEG decreases during propofol induced general anaesthesia. PLoS One 2015; 10: e0133532.

58. Mirsadeghi M, Behnam H, Shalbaf R, Jelveh Moghadam H. Characterizing awake and anesthetized states using a dimensionality reduction method. J Med Syst 2016; 40: 13.

59. Shalbaf R, Behnam H, Jelveh Moghadam H. Monitoring depth of anesthesia using combination of EEG measure and hemodynamic variables. Cogn Neurodyn 2015; 9: 41-51.

60. Schneider G, Jordan D, Schwarz G, Bischoff P, Kalkman CJ, Kuppe H, et al. Monitoring depth of anesthesia utilizing a combination of electroencephalographic and standard measures. Anesthesiology 2014; 120: 819-28. 